\title{
Medicalización de la vida (I)
}

\author{
Ramón Orueta Sáncheza, Coral Santos Rodríguez ${ }^{b}$, Enrique González Hidalgoc ${ }^{c}$, \\ Eva M ${ }^{a}$ Fagundo Becerrac ${ }^{c}$, Gemma Alejandre Lázaro ${ }^{a}$, Javier Carmona de la Morena ${ }^{a}$, \\ Javier Rodríguez Alcaláa, José $\mathrm{M}^{\mathrm{a}}$ del Campo del Campo ${ }^{\mathrm{b}}$, María Luisa Díez Andrés ${ }^{\mathrm{d}}$, \\ Natalia Vallés Fernández ${ }^{a}$, Teresa Butrón Gómez ${ }^{d}$
}

\begin{abstract}
${ }^{a}$ Médico de Familia, Toledo.
${ }^{\mathrm{b}}$ Médico de Familia, Albacete.

${ }^{\mathrm{c}}$ Médico de Familia,

Ciudad Real.

${ }^{\mathrm{a}}$ Médico de Familia,

Guadalajara.

Correspondencia: Ramón Orueta Sánchez, Centro de Salud Sillería, C/ Sillería s/n, 45001 - Toledo.

E -mail: roruetas@gmail.com.
\end{abstract}

Recibido el 5 de mayo de 2011.

Aceptado para su publicación el 12 de mayo de 2011.

\section{RESUMEN}

La medicalización de la vida es uno de los problemas que actualmente contribuyen a la masificación de las consultas del Médico de Familia y de los Servicios de Urgencias, provocando, a la vez, dificultades para proporcionar una atención de alta calidad y frustración en una buena parte de los profesionales.

Entendemos por medicalización el proceso de convertir situaciones que han sido siempre normales en cuadros patológicos y pretender resolver, mediante la medicina, situaciones que no son médicas, sino sociales, profesionales o de las relaciones interpersonales. La medicalización es un proceso continuo que se autoalimenta y crece de forma constante, facilitado por una situación en la que la sociedad va perdiendo toda capacidad de resolución y su nivel de tolerancia. Su origen es multifactorial, existiendo diversas causas y actores implicados (sociedad, medios de comunicación, industria farmacéutica, políticos, gestores y profesionales sanitarios), jugando el sector sanitario un papel fundamental en dicho proceso. Los profesionales sanitarios son, a la vez, actores y victimas de dicho proceso. Presentamos en este documento una reflexión sobre el proceso de medicalización de la vida y los factores intervinientes.

Palabras clave: Servicios de Salud, Procedimientos Innecesarios, Actitud frente a la Salud.

\section{ABSTRACT}

\section{Medicalisation of every-day life (I)}

Medicalisation of every-day life is one of the problems currently contributing to the massification of visits to the Doctors' surgeries or Hospital Emergency Departments, thus making it difficult to provide high quality healthcare and causing frustration for many health professionals.

Medicalisation is understood as being the process of turning normal human conditions into medical conditions and trying to resolve them through medicines. Such conditions are not medical, but social or professional conditions or having to do with interpersonal relationships. Medicalisation is an on-going process that feeds on itself and is steadily growing, aided by a situation in which society is losing all ability of resolution and tolerance. Its origin is multifactorial, and there are several causes and actors implicated (society, the media, the pharmaceutical industry, politicians, managers and health professionals), the health sector playing a key role in this process. Health professionals are both the actors and the victims of this process. We present a reflection on the process of medicalisation of every-day life and the intervening factors.

Key words. Health Services Misuse, Unnecessary Procedures, Attitude to Health.

\section{INTRODUCCIÓN}

La atención a la salud ha sido una prioridad en todas las sociedades desde que estas existen como tales y esta prioridad sigue presente en la actualidad, habiendo acaecido únicamente una evolución en los aspectos en los que se centra dicha atención, la forma de actuar sobre los mismos o la distribución de la atención entre las distintas capas sociales.

A lo largo de la historia, y actualmente en los países pobres, los esfuerzos prioritarios se han centrado en la obtención de los recursos básicos para la vida, como son el disponer de los alimentos suficientes para una alimentación adecuada, el garantizar el acceso a agua potable, etc. Una vez garantizados éstos, la atención se dirige hacia las enfermedades de mayor incidencia y 
las de mayor repercusión sobre la morbimortalidad, siendo las enfermedades infecciosas el paradigma de procesos de atención prioritaria en estos países.

Por el contrario, en los países desarrollados, garantizados el acceso a las necesidades básicas y la atención a los procesos patológicos existentes, aparece una tendencia creciente a abarcar dentro del ámbito sanitario situaciones que hasta este momento eran consideradas ajenas al mismo. En ellos, la sociedad ha desplazado al campo médico la búsqueda de soluciones a problemas inherentes a la realidad subjetiva y social de las personas y la obsesión por una salud perfecta se ha convertido en un factor patógeno predominante y así "una persona sana es solamente un enfermo sin identificar".

La denominada paradoja de la salud, existente tanto en la sociedad como a nivel individual en los países desarrollados, hace referencia a la constatación de que cuanto mayor es la situación objetiva de salud más son los problemas de salud declarados, y que cuanto mayores son los recursos empleados en salud mayor es la proporción de personas que se sienten enfermos.

La definición sobre salud de la OMS de 1946, que considera a ésta de forma utópica y subjetiva como algo más que la ausencia de enfermedad y la eleva al estado de bienestar absoluto, contribuye a la incorporación al ámbito médico de nuevas situaciones y problemas.

En la actualidad, situaciones de la vida como el cansancio o la frustración y variantes de la normalidad como la calvicie o la fealdad están siendo consideradas como enfermedades y, en muchos casos, sometidas a tratamiento farmacológico o incluso quirúrgico. Ni las propias etapas de la vida escapan a esta propensión a medicalizar, y así se tiende a someter al control médico el nacimiento, la adolescencia, la menopausia, etc.

En el momento actual el derecho a la salud esta convirtiéndose en un peligroso objeto de consumo, y no estamos lejos de un horizonte indeseable en el que la sociedad viva de manera insana por su desmedida preocupación por la salud. Estamos asistiendo a la generación de una dependencia de la medicina que incapacita a las personas para cuidarse a sí mismas y una disminución de la tolerancia al sufrimiento y al malestar.
Es urgente una reflexión colectiva sobre hacia dónde nos conduce la ruta trazada, las consecuencias de la medicalización de la vida y sobre qué tenemos que hacer los distintos actores implicados para reconducir dicha situación.

La Sociedad Castellano-Manchega de Medicina de Familia y Comunitaria (SCAMFYC) es conocedora de esta realidad y desde hace años está trabajando en aspectos relacionados con el tema. Fruto de este trabajo son los documentos "Uso Racional del Medicamento" (2003), "Nuevas Demandas de la Población" (2005) y "Uso Racional de los Recursos" (en elaboración).

A través del presente documento, SCAMFYC trata de reflexionar sobre el problema de la medicalización mediante el análisis de las causas y factores implicados en su desarrollo y la propuesta de acciones encaminadas a paliar dicha problemática.

\section{DEFINICIÓN DEL PROBLEMA}

El término medicalización es un neologismo que no es recogido en el Diccionario de la Real Academia Española de la Lengua, pero que hace referencia a una realidad actual que los profesionales reconocen con una significación propia.

En la década de los sesenta del pasado siglo, Meador acuñó el primer término que puede considerarse como precursor del término medicalización, al hablar de la "no enfermedad" y su atención, entendiéndose como tal aquel problema humano que es definido por alguna instancia como condición médica, pero para el que se obtendrían mejores resultados si no fuera considerado como tal.

Actualmente, la definición más aceptada sobre medicalización es la propuesta por Kishore, que la define como la forma en que el ámbito de la medicina moderna se ha expandido en los años recientes y ahora abarca muchos problemas que antes no eran considerados como entidades médicas y para los que la medicina no dispone de soluciones adecuadas ni eficaces. Quizás pueda ser más clara la definición que considera la medicalización como la conversión en procesos patológicos de situaciones que son y han sido siempre completamente normales y el pretender resolver mediante la medicina situaciones que no son médicas sino sociales, profesionales o de las relaciones interpersonales: 
- Se considera necesario el control médico de ciertas etapas de la vida (adolescencia, menopausia, envejecimiento, etc.).

- Problemas personales/sociales pasan a considerarse como problemas médicos (tristeza, duelo, síndrome postvacacional, etc.).

- Factores de riesgo pasan a considerarse como auténticas enfermedades (osteoporosis, dislipemia, etc.).

- Situaciones o cuadros poco frecuentes pasan a ser considerados, de forma artificial, como situaciones o cuadros frecuentes (disfunción eréctil, disfunción sexual femenina, etc.).

- Síntomas o cuadros leves son elevados, de forma artificial, a indicadores de cuadros graves (colon irritable, síndrome premestrual, etc.).

En una encuesta realizada a iniciativa de la revista British Medical Journal en 2002 sobre las principales "no enfermedades" que han pasado al control del campo médico destacaron: envejecimiento, alteraciones capilares (calvicie y canas), fealdad, celulitis, resaca e infelicidad, aunque otro estudio publicado con anterioridad en la misma revista puso en evidencia que existía cierta discrepancia entre profesionales y no profesionales a la hora de considerar o no, como enfermedad, algunas circunstancias o procesos.

\section{EL PROCESO DE MEDICALIZACIÓN}

El proceso de medicalización de un problema o situación no es algo instantáneo que acontece en un momento dado, sino que se produce de forma paulatina a lo largo de un periodo de tiempo, generalmente prolongado, y requiere de una serie de condiciones o pasos:

1. La situación, el sufrimiento o el problema debe ser considerado como algo anormal por parte de la Sociedad y, por lo tanto, precisa de una solución o abordaje por parte de algún sector de dicha Sociedad. La consideración de anormalidad, en general, es establecida por colectivos sociales poderosos (como la propia industria farmacéutica, que se ofertará más tarde para solucionar el problema), mientras que suelen ser los sectores sociales más débiles los que quedan englobados en dicha categoría. En muchos de los casos el problema no tiene nada que ver con una enfermedad, aunque la medicina pueda dar una solución (un ejemplo es la cirugía estética, que da respuesta a un sufrimiento que no es causado por ninguna enfermedad).

2. Los sectores encargados tradicionalmente de solucionar dicha situación de anormalidad no dan respuesta a la misma, o dan una respuesta insuficiente $\mathrm{o}$ no adecuada a las demandas sociales, por lo que la Sociedad rechaza estas respuestas y excluye o margina estos sectores tradicionales. Esta percepción de que la respuesta recibida es inadecuada puede verse influida por sectores que pueden verse beneficiados por este sentimiento.

3. Se inicia un proceso de búsqueda de nuevos sectores que den respuesta a las demandas no satisfechas. Aunque dicha búsqueda suele incidir sobre el sector sanitario, también puede realizarse en otros ámbitos.

4. Los distintos actores implicados en el proceso de asistencia sanitaria (profesionales, gestores, legisladores, etc.) deben aceptar que dicha situación pase a formar parte de su campo de actuación. Esta aceptación puede verse influenciada por presiones externas $y$, aunque siempre los objetivos asistenciales y humanitarios de la medicina se han visto influidos por aspectos políticos, económicos y de control social, éstos han ganado peso en las últimas décadas.

5. El sector sanitario busca soluciones para dar respuesta a los nuevos retos planteados. Con frecuencia, la solución aportada precisa de la realización de pruebas diagnósticas y la instauración de tratamiento (farmacológico, quirúrgico, etc.), generándose el fenómeno de la medicalización

6. Con frecuencia el círculo se cierra porque las actuaciones médicas implantadas no dan respuesta a los problemas, lo que genera mayor incertidumbre y aumento de la inseguridad. Como consecuencia, se generan nuevas situaciones que requieren de nuevas respuestas y éstas llegan, nuevamente, desde el sector sanitario. 


\section{FACTORES CAUSALES O INTERVINIENTES}

La progresiva medicalización de la vida es un problema de origen complejo en el que intervienen muy diversos factores de índole social, cultural, económico, sanitario, etc. Conocer y analizar estos factores facilita la toma de decisiones encaminadas a solucionar dicha situación.

Con carácter puramente expositivo (la mayoría de los factores podrían incluirse en varios de los apartados), estos factores pueden dividirse en función de su relación con la sociedad, los medios de comunicación, las instituciones político-sanitarias, los profesionales y la industria farmacéutica.

\section{Sociedad}

Sus creencias y valores van a determinar sus necesidades sentidas y sus expectativas.

- Cambios en el concepto salud/enfermedad. La medicina está vinculada al conjunto de la cultura de una sociedad y la trasformación de las concepciones médicas esta condicionada, a su vez, por las trasformaciones de las ideas de cada época. Sobre esta base, como el concepto salud/enfermedad se elabora a nivel social y cultural, este concepto debe valorarse en cada contexto sociocultural específico. En el momento actual se está produciendo una redefinición de fenómenos que antes eran propios de otras esferas para incluirlos en la esfera sanitaria. Por otra parte, el incremento de las capacidades tecnológicas aumenta la sensibilidad de la identificación de patologías y reduce el umbral a partir del cual tratarlas, aumentando así la incidencia de las enfermedades. Además, el riesgo (la presencia de factores de riesgo) ha pasado de ser considerado como una probabilidad de enfermar a ser considerado como una enfermedad, siendo los fármacos preventivos un mercado al alza. Todo ello hace que, cada vez con mayor frecuencia, se incluyan como enfermedad situaciones que antes eran consideradas de otro ámbito, aspectos meramente estéticos, síntomas y signos banales e inespecíficos, factores de riesgo, etc.

- Aumento del valor de la salud en la sociedad. Este aumento de valor se debe, en gran parte, a distintos aspectos negativos, como una mayor percepción de vulnerabilidad, mayor miedo a la enfermedad y a la muerte, etc. Sin poder negar el valor de la salud, éste no puede llevarse a un nivel en el que la sociedad haga negación del sufrimiento y de la enfermedad, lo que conlleva que, como dice una frase célebre, "la sociedad está enfermando por no enfermar".

- Acceso a la información. Uno de los mayores cambios sociales acontecidos en las últimas décadas es el mayor acceso a la información, e Internet ha supuesto un hito en este sentido. Los temas sanitarios no son ajenos a esta realidad. El problema surge no de la información en sí misma, sino de la calidad de la misma. La falta de rigor existente en diferentes fuentes, con Internet a la cabeza, ocasiona errores y sesgos importantes que aumentan expectativas, generan autodiagnósticos, etc. Algún autor denomina "malicia sanitaria" dar información a través de medias verdades, extrapolaciones atrevidas, interpretaciones sesgadas, simplificación del problema, etc. Aunque algunos aspectos se mencionan con más detalle en los apartados dedicados a la industria farmacéutica y los medios de comunicación, solo recordar que una parte no desdeñable $(20 \%)$ de los gastos de la industria farmacéutica en publicidad va dirigido a la población y que en muchas ocasiones esta promoción no aparece como anuncios sino como publirreportajes, "campañas de concienciación", etc.

- Capacidad de decisión. Aunque el concepto es considerado como un aspecto positivo, debe tenerse en cuenta que la población solo actúa y elige en libertad cuando dispone de información veraz y completa, pero es sabido, como se comenta en el apartado anterior, que generalmente recibe información parcial y sesgada y esto determina que su capacidad de decisión esté muy influida.

- Secularización de la sociedad. El declive de la función religiosa en el consuelo y afrontamiento de los problemas ha originado que la búsqueda de soluciones se realice a otros niveles y los servicios sanitarios ocupan un lugar destacado en esta búsqueda.

- La medicina como parte del mercado de consumo. El aumento del nivel de vida en 
los países desarrollados lleva aparejado un aumento del consumismo. El mercado fomenta la demanda de medicina y tecnología médica, que han pasado a ser bienes de consumo. Los medicamentos $y$, en menor medida las pruebas complementarias, son el objeto material de cambio en la relación mercantil entre el médico y la población. El mercado anima, además, a que el paciente tenga capacidad de elegir no solamente entre las alternativas diagnósticas o terapéuticas apropiadas para su proceso, sino también para solicitar aquellos que no están indicados. Es lo que algunos autores denominan "doctor shopping". En resumen, la enfermedad se ha convertido en un producto industrial alimentado por el deseo de estar sano.

- Fascinación por la tecnología. Existe el sentimiento creciente de que cualquier prueba es mejor que la simple valoración médica o de que la técnica última en aparecer es por definición mejor que las existentes previamente, y esto comporta pérdida de ciertos valores como la relación médico-paciente o el componente socio-cultural de los procesos.

- Expectativas ilimitadas. Las expectativas, como todas las creencias y valores, vienen determinadas por factores externos e internos del sistema y están sometidas a cambios permanentes, siendo los profesionales una de las principales fuentes de dichas creencias/ expectativas. La Sociedad, pero también los gestores y los profesionales (aunque en menor medida), espera resultados ilimitados de la medicina: pruebas para todo, curación de todas las enfermedades, prevención sin límites, etc. La tecnificación y los avances de la medicina han creado expectativas irreales llegándose a la idea utópica de que la ciencia encontrará la solución a todas las enfermedades y problemas. Por este motivo, no se acepta que no existan soluciones o tratamientos para todas las patologías.

- Disminución de los niveles de tolerancia. La Sociedad actual ha experimentado una reducción del nivel de tolerancia al dolor y al sufrimiento y requiere de la atención sanitaria para situaciones que antes eran aceptadas y resueltas sin necesidad de recurrir a los profesionales sanitarios. Es lo que algunos autores denominan "tolerancia cero". Al solicitar atención, se produce una medicalización de dichas situaciones y esto genera mayores expectativas y menor tolerancia, cerrando un circulo de perpetuación de la situación.

- Búsqueda de soluciones inmediatas. Muy unida a la disminución del nivel de tolerancia está la búsqueda inmediata de soluciones, lo que condiciona la consulta nada más aparecer los síntomas. Se exigen todo tipo de pruebas diagnósticas que permitan un diagnóstico inmediato y se solicitan tratamientos que resuelvan rápidamente el cuadro. Todo ello genera que en las fases iniciales de procesos (con síntomas inespecíficos y variables), y ante la presión de la población, se prescriban tratamientos que con alta probabilidad van a resultar ineficaces y ello genera nuevas consultas y nuevos tratamientos.

- Disminución de la capacidad de auto-cuidados. Actualmente se tiende a considerar que el sistema sanitario debe ser el garante de todo tipo de cuidados, por lo que se va perdiendo la capacidad de que la persona genere cuidados para sí misma. El acceso a la información, la capacidad de desarrollo individual o el aumento del consumo tienen aspectos muy positivos, pero a la vez han generado unas expectativas desmesuradas, una disminución de la seguridad y de la capacidad de contención de problemas y un aumento del miedo a enfermar, y todo ello ha conducido a una pérdida de la capacidad de autocuidados y a un aumento del consumo de servicios sanitarios. Dos ejemplos claros en este sentido son la preferencia de los medicamentos (ayuda externa) frente a los cambios en el estilo de vida (esfuerzo personal) y el papel denostado que ha adquirido la automedicación, de la que se han destacado únicamente los aspectos negativos, cuando es una herramienta útil para la solución de múltiples cuadros menores.

- Reducción de las redes informales de cuidados. Distintos cambios sociales acontecidos en las últimas décadas (reducción del tamaño familiar, cambios en la estructura familiar, incorporación de la mujer al mercado laboral, etc.) han ocasionado que los recursos informales se hayan visto reducidos y que su lugar esté 
siendo ocupado, cada vez en mayor proporción, por los cuidados formales, con la consiguiente tendencia a la medicalización.

- Escasa educación sanitaria de la población. Aunque buena parte de la población tiene un acceso prácticamente ilimitado a la información, la parcialidad de ésta, la baja calidad general y la falta de pensamiento crítico unido a un sentimiento de exigencia por los derechos personales, muy por encima de las responsabilidades colectivas, repercuten en un escaso nivel educativo sanitario, proporcionando al individuo muy escasos recursos para enfrentarse tanto a los problemas personales como colectivos, trasladando esta responsabilidad al sistema sanitario.

\section{Medios de Comunicación}

Conocidos como "el cuarto poder", juegan un importante papel en la transmisión de la información a la población y, por ello, son moduladores de las creencias y expectativas de la sociedad.

- Fuente de información sanitaria. Los medios de comunicación ocupan un lugar destacado en cuanto a la información sanitaria recibida por la población. Representan, tras los profesionales, el segundo lugar como fuente de información sanitaria. Ello es debido a que un porcentaje importante de las noticias que transmiten abordan temas relativos a la salud.

- Poder mediático. Algunos trabajos han evidenciado la relación directa entre la aparición de una noticia médica en los medios de comunicación y la utilización de los servicios sanitarios por dicho motivo.

- Sesgos de información. Los medios de comunicación tienen el derecho y el deber de informar, pero el concepto de noticia de interés se relaciona con la novedad, con lo deseado, con lo excepcional. Ello hace que la tendencia sea a informar sobre métodos diagnósticos infalibles $y$ terapéuticos con eficacia segura. Este sesgo en la información genera expectativas irreales que contribuyen a la medicalización.

- Información versus publicidad. En diversas ocasiones lo que es mera publicidad aparece camuflada como información bajo la fórmula de publirreportaje, y a los lectores no siempre les resulta sencillo identificar que aquello es un mensaje publicitario pagado por una empresa con interés en su promoción.

- Promoción de alimentos "saludables". Una parte importante de la información y de la publicidad aborda el tema de alimentos "saludables" (por ejemplo, cereales que regulan el ritmo intestinal, yogures con probióticos, etc.), de los que en muy pocos casos existen estudios que demuestren esas supuestas virtudes destacadas. Ello contribuye a medicalizar una parte importante de la vida como es la alimentación y, además, generan falsas expectativas que crean decepción al no verse satisfechas.

\section{Instituciones Político-Sanitarias}

Los políticos y los gestores son los responsables de establecer las estrategias a seguir y definir la cartera de servicios que se presta a la población, y estos aspectos pueden jugar un importante papel en la medicalización de la vida.

- Modelo universal, accesible y abierto. Nuestro modelo sanitario permite el acceso de todas las personas a todos los servicios, de forma libre y gratuita, y en todo momento. Estas características representan algunos de los principales puntos positivos y fuertes de nuestro sistema sanitario, pero tienen como connotación negativa que facilitan la medicalización de la sociedad.

- Servicios y organizaciones basados en demandas y no en necesidades. Diversos servicios, organizaciones, etc. se han creado y estructurado en función de demandas de la población, y estas demandas, que con frecuencia son fomentadas por sectores con intereses específicos, no siempre guardan relación con las necesidades reales de la sociedad ni existen intervenciones eficaces para resolverlas. Un ejemplo en este sentido son los Servicios de Urgencias en Atención Primaria. Fueron creados con el objetivo de atender situaciones de urgencia que no admiten demora y que han pasado a denominarse Puntos de Atención Continuada, con el mensaje de que se acude a los mismos siempre que se considere oportuno, con independencia de la urgencia real o no de su situación. Esto ha llevado a su masificación y, como consecuencia, al aumento 
de su dotación humana y material, y todo ello utilizándose como pretexto la demanda de la población.

- Políticas paternalistas y acríticas. Aunque el artículo 43 de la Constitución Española hace al Estado garante y responsable de la protección de la salud de la población, esto no implica que no deban realizarse políticas de fomento de la autonomía y del autocuidado y de dimensionar correctamente las expectativas y las responsabilidades individuales en materia de salud. El sistema sanitario hace suyas funciones que antes quedaban fuera de su ámbito de actuación y, además, no aclara las limitaciones del mismo en cuanto a recursos o resultados, facilitando la creación de expectativas alejadas de la realidad. Además, el sistema se preocupa de mejorar su eficiencia a través de medidas organizativas y de gestión, pero sin apenas valorar el objetivo (hacia dónde vamos y qué pretendemos conseguir). Por otra parte, los políticos, y en menor medida los gestores, tratan de evitar la toma de decisiones conflictivas que puedan hacer disminuir su popularidad y tienden a poner en marcha actuaciones de gran aceptación popular con independencia de su efectividad. En base a ello, se realizan intervenciones sanitarias para todo tipo de problemas: olas de calor, dificultades educativas, inadecuaciones laborales, etc.

- Papel preponderante de la Atención Hospitalaria frente a la Atención Primaria. Aunque no hay ponencia o discurso donde no se diga que la Atención Primaria es el eje del sistema, esto casi nunca se ve reflejado ni en las grandes decisiones políticas ni en las decisiones de gestión directa. Esta perdida de rol principal y de recursos hace que la atención prestada en el primer nivel asistencial sea menos resolutiva y más dependiente de otros niveles asistenciales. La población lo interioriza como una falta de valor de la Atención Primaria y va teniendo la percepción de que precisa la realización de pruebas complementarias ante cualquier dolencia o que cualquier cuadro deba ser valorado por el especialista correspondiente, y todo ello contribuye a la medicalización.

- Creación de sistemas/estructuras paralelos. El propio sistema crea servicios, tanto a nivel de atención primaria como hospitalaria, que compiten entre sí para la atención de enfermedades (por ejemplo, equipos de atención domiciliaria para pacientes terminales) e incluso para no-enfermedades (por ejemplo, consulta de atención al adolescente). Ello genera, además de una pérdida de eficiencia de los recursos, pérdida de visión integral de la persona, mensajes discordantes que aumentan la incertidumbre y la angustia o mayores expectativas de la población, aumento, en resumen, de la medicalización.

- Burocratización sanitaria de la Sociedad. La incorporación al campo sanitario de burocracia externa al mismo (justificantes de ausencias escolares, impresos para vacaciones de la tercera edad, parte de descanso maternal, etc.) contribuye a fomentar la creencia de que dichas situaciones son situaciones dependientes a dicho sistema y se crean nuevas expectativas respecto al mismo.

- Los Organismos reguladores/controladores y sus relaciones. En diversas ocasiones existen dudas de la independencia de los Organismos o Instituciones reguladores/controladores. Es conocida, por publicada, la participación de la FDA en la defensa de algunas compañías farmacéuticas, pero también son conocidas las relaciones en nuestro entorno, como que la reciente Ley de garantías y uso racional de los medicamentos y productos sanitarios, en el apartado de aportaciones por ventas, establezca una graduación que se base en criterios de política industrial y no sanitarios.

- Expansión acrítica de la medicina preventiva centrada en el individuo. La prevención es popular y todo el mundo acepta como obvio el "más vale prevenir que curar", lo que facilita la implementación de medidas preventivas (cribados, vacunas, etc.), con un dudoso balance entre riesgo y beneficio. Esta expansión contribuye a la medicalización de la sociedad y crea falsas expectativas sobre la posibilidad de evitar todos los riesgos. Este modelo es con frecuencia dogmático y culpabiliza a aquellos profesionales o usuarios que disienten.

\section{Profesionales}

Los profesionales sanitarios ven de forma directa 
en su práctica clínica diaria las consecuencias de la medicalización, pero a su vez tienen un importante papel en la aparición de dicho fenómeno.

Varios de los factores comentados en el apartado de políticos-gestores (paternalismo, atención basada en la demanda, etc.) son igualmente válidos en este apartado dedicado a los profesionales.

- Grupo fundamental en la medicalización. Los profesionales constituyen el grupo fundamental de los actores implicados, ya que tienen la capacidad/poder de determinarquédiagnósticos establecen y qué actuación terapéutica realizan. El resto de grupos implicados (sociedad, medios de comunicación, políticos, etc.) pueden fomentar la medicalización, pero son los profesionales quienes finalmente la llevan a cabo. Pero, aún siendo los responsables finales, no se debe olvidar que están sometidos a una doble presión científico-técnica y social que les induce a la misma, siendo en múltiples ocasiones los únicos que escuchan y aportan algún tipo de actuación a diversas situaciones, aunque éstas no sean efectivas.

- Pérdida de la visión multifactorial de la enfermedad. La formación médica se centra básicamente en la esfera biológica de los procesos y esto condiciona que exista una tendencia creciente, apoyada por la tecnología, de centrar el origen de todos los procesos en su vertiente biológica, dejando a un lado los factores psicológicos y sociales implicados.

- Parcelación de la atención. Existe una creciente superespecialización de la medicina, lo que fragmenta la atención prestada. Cada persona acaba siendo atendida por diversos profesionales. Esto determina que se genere un sobredimensionamiento de los problemas, lo que generalmente deriva en un mayor número de controles realizados, de pruebas complementarias solicitadas, en muchas ocasiones simples repeticiones de la misma prueba, y de medicamentos prescritos con los riesgos y problemas que todo ello puede generar.

- Sobrecarga de trabajo. Uno de los grandes problemas sin resolver en la asistencia sanitaria, especialmente en Atención Primaria. Este exceso de demanda determina que el tiempo disponible para cada paciente sea escaso, siendo difícil establecer, en el escaso tiempo disponible, las verdaderas necesidades y demandas de los pacientes, sus conocimientos sobre el tema y sus posibilidades de autocuidados, traduciéndose todo ello en la realización de pruebas y la prescripción de tratamientos innecesarios que fomentan la medicalización. No puede dejar de comentarse en este apartado que, en ocasiones, la sobrecarga de trabajo es la razón esgrimida para no realizar algunas actividades y de justificar la forma de realización de otras.

- Trabajo con incertidumbre. Todos los profesionales, pero especialmente los de Atención Primaria, trabajan con incertidumbre debido a diversas circunstancias: fases iniciales de la enfermedad (signos y síntomas con gran indefinición y variabilidad), relaciones dudosas de causalidad, escasos estudios sobre la efectividad de las intervenciones, etc., y ello genera dudas y ansiedad, que contribuyen al empleo de fármacos de forma defensiva y esto, a su vez, contribuye a la medicalización. En una atención fuertemente burocratizada algunos profesionales han podido sentirse cómodos ante una responsabilidad protegida entre "papeles" (partes, recetas, informes, pruebas complementarias, derivaciones, etc.). En esta situación el manejo de la incertidumbre, como un hecho natural propio del proceso saludenfermedad y característico del primer nivel asistencial, puede resultar un lujo.

- Complejidad para establecer el límite entre la normalidad y la anormalidad. Muy unido al punto anterior, los profesionales no siempre pueden establecer con absoluta certeza qué persona está sana y cuál enferma, qué acontecimientos son normales y cuáles no, y ello determina dudas que conducen a una mayor petición de pruebas complementarias y a la instauración de tratamientos innecesarios. Un ejemplo citado por varios autores de esta dificultad de establecer los límites entre sano y enfermo es la clasificación DSM-IV de trastornos mentales, que identifica como tales situaciones el trastorno de ansiedad por desaparición, los problemas paternofiliales o los problemas religiosos y/o espirituales. 
- Conversión a enfermedad de los factores de riesgo. Existe una tendencia creciente a tratar como enfermedad lo que no son más que factores de riesgo, convirtiendo en enfermos personas que únicamente tienen aumentado su riesgo de enfermar y sin que en muchos de los casos existan evidencias del beneficio de actuar sobre dichos factores.

- Dificultad en el empleo del rigor científico. No existen estudios que demuestren la validez de muchos de los métodos diagnósticos existentes ni sobre la eficacia o efectividad de un alto número de tratamientos que con frecuencia se prescriben. Además, es conocido que existe un sesgo en los estudios publicados en las revistas científicas y en las recomendaciones de expertos, ocasionado por diversos motivos. Entre ellos destaca la intervención de la industria farmacéutica, pues una parte importante de la investigación está financiada por ella. Las revistas científicas cubren parte de sus presupuestos gracias también a dicha industria (anuncios, separatas de los ensayos clínicos financiados por ella, etc.), e incluso muchos de los grupos de expertos que emiten recomendaciones existen gracias al patrocinio de la misma. En este apartado también pueden considerarse los estudios en subgrupos artificiales ad-hoc y los megaensayos para obtener significación estadística en mínimas diferencias, en los que las conclusiones obtenidas (que eran las esperadas) son las que han determinado la metodología del mismo.

- Sobreutilización de las nuevas tecnologías. Quizás, en parte influidos por la dificultad en establecer el límite entre lo normal y lo patológico y por la falta de evidencias científicas sobre las actuaciones recomendables en diversas circunstancias, los profesionales tienden a utilizar con frecuencia las nuevas tecnologías, aunque no se conozca con certeza su validez y éstas aumenten la sensibilidad de la identificación de alteraciones de la normalidad y reduzcan el umbral a partir del cual deben abordarse médicamente. Se pueden incluir en este apartado las pruebas de detección genética, que están otorgando la etiqueta de enfermo a personas por el solo hecho de tener una alteración genética que aumenta la probabilidad de sufrir una enfermedad en el futuro.

- Tendencia a la innovación terapéutica. España ocupaunodelos primerospuestosanivelmundial en el empleo de nuevos fármacos, aunque un alto porcentaje de estas nuevas moléculas no aporten avances destacables respecto a las ya existentes (medicamentos "me-too"). Esta oferta constante de nuevos tratamientos crea un aumento de las expectativas que casi nunca se ve compensado.

- Puesta en marcha de actividades sin evidencia de su utilidad. Con frecuencia se ponen en marcha distintas actividades con el objetivo plausible de dar nuevos servicios a la población, pero para las que no existen evidencias de su eficacia/efectividad y que fomentan la medicalización al generar la idea de que son actividades necesarias. Además, al existir falta de resultados se genera mayor ansiedad e incertidumbre y nuevas consultas. Ejemplos de ello son muchas de las actividades realizadas dentro del programa del niño sano (conocido de forma sarcástica como programa del niño "crónicamente sano") o de los reconocimientos de empresa. Algunos autores hablan de obstinación terapéutica, entendiendo como tal la realización de tratamientos o intervenciones que no aportan beneficio para el paciente, abarcando más conceptos que el clásico de actuaciones en el paciente terminal.

- Medicina defensiva. Probablemente en relación con varios de los puntos ya comentados (sobrecarga asistencial, trabajo con incertidumbres, falta de evidencias en muchas situaciones, etc.), junto con el temor a posibles denuncias, existe un tendencia creciente a realizar una medicina defensiva que se traduce en más pruebas complementarias y más tratamientos "por si acaso" que contribuyen al proceso de medicalización de los problemas.

- Inercia "medicalizadora" y "farmacolizadora", entendiendo como tal la tendencia inmediata a realizar una intervención, generalmente solicitar alguna prueba complementaria o pautar un tratamiento farmacológico, cada vez que una persona consulta, sea cual sea el motivo de la misma. También puede quedar englobado 
en este apartado el seguimiento inadecuado de procesos con tendencia a aumentar dosis o añadir nuevos fármacos ante la persistencia de síntomas, signos o alteraciones en las pruebas complementarias, sin verificar si dicha persistencia puede deberse a situaciones como el incumplimiento terapéutico.

- Prescripción inducida. Si bien es cierto que el problema de medicalización en general, y farmacolización en particular, es común a todos los sectores sanitarios, no es menos cierto que en la Atención Hospitalaria este problema se ve acrecentado. Esto repercute negativamente en la Atención Primaria, ya que una parte importante de su prescripción es inducida (30-45\%), y porque, aunque existe desacuerdo en una parte importante de esta prescripción inducida (15-30\%), es difícil de solventar.

- Limitaciones de las recomendaciones de Sociedades Científicas y grupos de expertos. Vienen dada por dos razones. En primer lugar, la falta de evidencias que sostengan las actuaciones en todas las situaciones a las que se deben enfrentar los clínicos. En segundo lugar, y como ya se ha mencionado en el punto anterior, en ocasiones, las Sociedades Científicas y los grupos de expertos, cuyo importante papel está fuera de discusión, ven limitada su capacidad por las relaciones existentes, económicas y no económicas, con las Instituciones o con la industria farmacéutica, y ello puede ocasionar sesgos en sus recomendaciones.

- Relaciones con la industria farmacéutica. Los profesionales mantienen con frecuencia relaciones con la industria farmacéutica que sin duda condicionan algunas de sus decisiones y pueden suponer un aumento de la prescripción de fármacos que favorece la medicalización de algunas circunstancias.

\section{Industria farmacéutica}

La industria farmacéutica representa otra parte de los factores implicados en la medicalización de la vida, pero, aunque su papel es conocido, no por ello se debe caer en la focalización de toda la responsabilidad. Es sin duda una pieza importante del puzzle, pero únicamente una pieza del mismo.
- La industria farmacéutica como empresa. Las empresas farmacéuticas, como cualquier otra, buscan obtener los máximos beneficios. Este es un objetivo lógico y lícito. Su problema es que la obtención de este objetivo se realiza a través de estrategias que no siempre resultan beneficiosas para la salud de la población y contribuyen a su medicalización.

- Búsqueda de mercado. Se busca aumentar el mercado de un medicamento a través de varias vías: nuevas indicaciones, ajustes de los objetivos terapéuticos (reducir la cifra de un valor considerada como normal supone más personas tratadas y con mayores dosis), presentación como innovación de lo que únicamente supone la introducción de pequeñas modificaciones moleculares (isómeros, presentaciones de liberación retardada, etc.), etc., y varias de estas estrategias contribuyen claramente a la medicalización. En ocasiones, la industria presta escaso interés en encontrar nuevos fármacos para enfermedades existentes y centra parte de sus esfuerzos en dar salida a fármacos existentes a través de la creación de nuevas enfermedades.

- Investigación sesgada. Una parte importante de los avances en el campo médico, especialmente en su faceta terapéutica, se han producido gracias a la investigación realizada o patrocinada por la industria farmacéutica, pero esto es un arma de doble filo. Al financiar y promocionar gran parte de los ensayos clínicos está en condiciones de influir en el tipo y objetivos de las investigaciones desarrolladas, lo que determina en gran medida la "evidencia disponible" (solo existe evidencia de lo que se investiga/publica y no existe evidencia de lo que no se investiga o no se publica). Esto influye en los profesionales que tratan de hacer su trabajo correctamente a través del empleo de la evidencia disponible. Además, la investigación patrocinada por la industria tiende a ser más favorable a los productos de dichos laboratorios que los estudios independientes.

- Promoción de enfermedades. La industria dedica una parte importante de su presupuesto a la denominada "disease mongering" (promoción de enfermedades), que hace referencia al esfuerzo que realizarían las compañías 
farmacéuticas por llamar la atención sobre condiciones o enfermedades frecuentemente inofensivas, con objeto de incrementar la venta de medicamentos, mediante campañas publicitarias, visitadores médicos, estudios que intentan medicalizar cualquier dolencia, etc., pero esta inversión les resulta rentable. Existen distintas estrategias para conseguir este objetivo: tomar un síntoma común y hacerlo parecer el signo de una enfermedad importante, definir el porcentaje de población que padece un problema lo más ampliamente posible, usar sesgadamente la estadística, etc. El síndrome de hiperactividad en el niño, la disfunción sexual o el trastorno de ansiedad social son algunos de los ejemplos que han sido ampliamente comentados en la literatura científica.

- Promoción de medicamentos. Las empresas farmacéuticas invierten en el marketing de sus fármacos una parte destacada de su presupuesto y esta promoción se dirige tanto a los profesionales como a la población general. La parte más importante de la promoción $(80 \%)$ se dirige a los profesionales e Instituciones, realizándose habitualmente a través de visitas, pequeños obsequios (por ejemplo, libros), inscripciones a congresos, etc., pero en ocasiones la influencia es más sutil a través de formación sesgada (charlas de expertos seleccionados, pseudocongresos promocionales, etc.). Otra parte de la promoción se dirige directamente a la población $y$, aunque representa un porcentaje menor (aproximadamente un 20\% según alguna publicación), tiene un impacto importante, ya que genera opinión y demanda.

\section{BIBLIOGRAFÍA}

1. Angell M. Excess in the pharmaceutical industry. CMAJ. 2004; 171:1451-53.

2. Blech J. Los inventores de enfermedades. Barcelona: Destino; 2005.

3. Bordas JM, Casajuana J. Prescripción inducida en atención primaria: de la excusa pasiva a la gestión activa. FMC. 2000; 5:323-28.

4. Cabral JA. Nuevas tendencias de la medicalización. Ciencia \& Saude Colectiva. 2008; 13(supl):579-87.

5. Campbell EG, Russell LG, Mountford J, Miller LG, Cleary $P D$, Blumenthal D. A national survey of physician-industry relationships. N Engl J Med. 2007; 356:1742-50.
6. Campbell EG, Weissman JS, Ehringhaus S, Rao SR, Moy $B$, Goold SD. Institutional Academic-Industry relationship. JAMA. 2007; 298:1779-86.

7. Campbell EJM, Scadding JG, Roberts RS. The concept of disease. BMJ. 1979; 8:757-62.

8. Casino G. La Promoción de la Enfermedad. El País. 2006 (25/04/06). p. 39.

9. Davis P. Health care as a risk factor. CMAJ. 2004; 170:168889.

10. Díaz JL. El sufrimiento medicalizado. Cultura de los cuidados. 2008; 23:50-6.

11. Fernández MJ, Marquet $R$, Reguant $M$, Zaapter $F$. ¿Enfermos de salud?. Societat Catalana de Medicina Familiar i Comunitária. 2006. Disponible en: http://www. camfic.org.

12. Gérvas J. Malicia Sanitaria y prevención cuaternaria. Gac Med Bilbao. 2007; 104:93-6.

13. Gérvas J, Pérez-Fernández M. El auge de las enfermedades imaginarias. FMC. 2006; 13:109-11.

14. Gérvas J. Moderación en la actividad médica preventiva y curativa. Cuatro ejemplos de necesidad de prevención cuaternaria en España. Gac Sanit. 2006; 20(supl 1):12734.

15. Gottlieb S. A fith of Americans contact their doctor as a result of drug advertising. BMJ. 2002; 325:854.

16. Grilli R, Ramsay C, Minozzi S. Mass media interventions: effects on health services utilisation (Cochrane Review). In: The Cochrane Library. Inssue 4, 2002. Oxford: Update software.

17. Hijmans E, Pleijter A, Wester F. Covering Scientific Research in Dutch Newspapers. Science Comunication. 2003; 25(2):153-76.

18. Hofmann B. The technological invention of disease. J Med Ethics: Medical Humanities. 2001; 27:10-9.

19. Illich J. L'obsesion de la santé parfacte. Le Monde Diplomatique. 1999; 41:21.

20. Induces prescription in Barcelona's Primary Care Study Group (Institut Catalá de la Salut). Induced prescription in primary healthcare. Eur J Gen Pract. 1999; 5:49-53.

21. Kishore J. A dictionary of Public Health. New Dephi: Century Publications; 2002

22. Lumbreras B, Hernández-Aguado I. El entusiasmo por las pruebas diagnósticas: efectos en la salud y formas de control. Gac Sanit. 2008; 22(Supl 1):216-22.

23. Mainetti JA. La Medicalización de la Vida. Electroneurobiología 2006; 14:71-89.

24. Maglio I. La medicalización de la vida como causa de reclamos contra la practica médica. Medicina (Buenos Aires). 1998; 58: 548-53.

25. Marquet R. La obsesión por la salud. FMC. 2006; 13:5079.

26. Márquez-Calderón S. Los efectos negativos de las intervenciones preventivas basadas en el individuo. Gac Sanit. 2008; 22(Supl 1):205-15.

27. Marquez S, Meneu R. La medicalización de la vida y sus protagonistas. Ges Clinica y Sanitaria. 2003; 5:47-53.

28. Meador $\mathrm{C}$. The art and science of nondisease. $\mathrm{N}$ Engl $\mathrm{J}$ Med. 1965; 272:92-5.

29. Meneu R. Las "no enfermedades". AMF. 2005; 1:123-4.

30. Merino A, Miguel F, Sanz R. Yo medicalizo, tú medicalizas, él...; reflexiones y propuestas. AMF. 2005; 1:269-78.

31. Miguel F, Merino A, Sanz R, García A, Montero MJ, Maderuelo JA. Procedimientos operativos y técnicos en la medicalización de la prevención. Aten Primaria. 2009. 41:702-6.

32. Moynihan R, Doran E, Henry D. Disease mongering is now parto $f$ the global health debate. Plos Med. 2008; 5:e106. 
33. Moynihan R, Heath I, Henry D. Selling sickness: the pharmaceutical industry and disease mongering. BMJ. 2002; 324:886-91.

34. New York Times. Ethical Journalism: a handbook of values and practices for the News and Editorial Departaments. New York, 2004.

35. Pellechia M. Trenes in science coverage: a contect análysis of three US newspapers. Public Understanding of Science. 1997; 6:49-68.

36. Puig-Junoy J. La financiación y la regulación del precio de los medicamentos en el Sistema Nacional de Salud: cambios y continuidad. Gac Sanit. 2007; 21(1):1-4.

37. Pundik J. La medicalización de la infancia. ¿Cada vez más frecuente? FMC. 2009; 16:213-18.

38. Quintana O. Los objetivos de la medicina. Rev Calidad Asistencial. 2003; 18:132-35.

39. Reverte D. El negocio de la sanidad y la medicalización de la vida. Salud 2000. 2007; 112:8-16.

40. Rodríguez S. El proceso de medicalización y sus consecuencias. Entre la moral, el poder y el negocio. Intersticios. 2008; 2:71-85.

41. Schwitzer G, Mudur G, Henry D, Wilson A, Goozner M, Simbra $M$ et al. What are the roles and responsibilities of the media in disseminatinh health information? Plos Med. 2005; 2(7):e215.

42. Sen A. Health: perception versus observation. BMJ. 2002; 324: $860-1$.
43. Smith R. Why are doctors so unhappy?. There are probably many causes, some of them deep. BMJ. 2001; 322:107374.

44. Smith R. In search of "non-disease". BMJ. 2002; 324:8835.

45. Sociedad Castellano-Manchega de Medicina Familiar y Comunitaria. Nuevas demandas de la población. Scamfyc. 2005. Disponible en: http://www.scamfyc.org.

46. Sociedad Castellano-Manchega de Medicina Familiar y Comunitaria. Propuestas para mejorar el uso racional del medicamento y racionalizar el gasto farmacéutico. Scamfyc. 2003. Disponible en: http://www.scamfyc.org.

47. Tizon JL. De los procesos de duelo a la medicalización de la vida. Jano. 2006; 1818:58-60.

48. Vara AM. Como medicalizar la vida diaria: la creación de enfermedades o "disease mongering". Evid Actual Pract Amb. 2008; 11:130-2.

49. Zapater F. Hasta aquí hemos llegado. Los límites de la medicina. Rev Calidad Asistencial. 2006; 21:1-2.

50. Zapater F. nuevos pacientes y nuevas demandas. ¿Qué hacer?. En: Casajuana J. Gestión del día a día en el Equipo de Atención Primaria. Barcelona: SemFYC; 2007

51. Zapater F, Vila R. Habilidades para no medicalizar. FMC. 2008; 15:670-2. 